\title{
MOONLIGHT: A NEW LUNAR LASER RANGING RETROREFLECTOR INSTRUMENT
}

\author{
M. Garattini ${ }^{a, *}$, S. Dell' Agnello ${ }^{a}$, D. Currie $^{b}$, G. O. Delle Monache ${ }^{a}$, \\ M. Tibuzzi ${ }^{a}$, G. PAtrizi ${ }^{a}$, S. Berardi ${ }^{a}, \mathrm{~A}$. Boni ${ }^{a}, \mathrm{C} \mathrm{CANTONE}^{a}$, \\ N. Itaglietta ${ }^{a}, \mathrm{C} \cdot \operatorname{Lops}^{a}, \mathrm{M} . \mathrm{MAIEllo}^{a}, \mathrm{M} . \mathrm{Martini}^{a}, \mathrm{R} . \mathrm{Vittori}^{c}$, \\ G. Bianco ${ }^{d}$, R. MARCH $^{a}$, G. Bellettini ${ }^{a}$, R. TAUraso ${ }^{a}$ \\ ${ }^{a}$ Istituto Nazionale di Fisica Nucleare - Laboratori Nazionali di Frascati, Via E. Fermi 40, 00044 Frascati \\ (Roma), Italy \\ ${ }^{b}$ Department of Physics, University of Maryland (UMD), College Park, MD 20742 and NLSI (NASA Lunar \\ Science Institute), USA \\ ${ }^{c}$ European Space Agency (ESA-HSO) and AMI - Aeronautica Militare Italiana \\ ${ }^{d}$ Centro di Geodesia Spaziale G.Colombo (ASI-CGS), Matera, Italy \\ * corresponding author: marco.garattini@roma1.infn.it
}

\begin{abstract}
Since 1969 Lunar Laser Ranging (LLR) to the Apollo Cube Corner Reflector (CCR) arrays has supplied several significant tests of gravity: Geodetic Precession, the Strong and Weak Equivalence Principle (SEP, WEP), the Parametrized Post Newtonian (PPN) parameter $\beta$, the time change of the Gravitational constant $(G), 1 / r^{2}$ deviations and new gravitational theories beyond General Relativity (GR), like the unified braneworld theory (G. Dvali et al., 2003). Now a new generation of LLR can do better using evolved laser retroreflectors, developed from tight collaboration between my institution, INFN-LNF (Istituto Nazionale di Fisica Nucleare - Laboratori Nazionali di Frascati), and Douglas Currie (University of Maryland, USA), one of the fathers of LLR. The new lunar CCR is developing and characterizing at the "Satellite/Lunar laser ranging Characterization Facility" (SCF), in Frascati, performing our new industry standard space test procedure, the "SCF-Test"; this work contains the experimental results of the SCF-Test applied to the new lunar CCR, and all the new payload developments, including the future SCF tests. The International Lunar Network (ILN) research project considers our new retroreflector as one of the possible "Core Instruments".
\end{abstract}

KEYWORDS: Lunar Laser Ranging, general relativity, gravitation, equivalence principle, geodetic precession, gravitational constant variation, PPN parameters, APOLLO station, International Lunar Network, Cube Corner Retroreflector.

\section{INTRODUCTION}

The time of flight measurement of a laser pulse sent by a station on the Earth in the direction of a laser retroreflector deployed on the lunar surface, and sent back again to the station, is commonly know as Lunar Laser Ranging and is the most accurate and cheapest distance measurement in space. Generally realized in fused silica, this special kind of laser mirror is a solid CCR, which, assembled in passive, maintenance-free, light-weight Laser Retroreflector Arrays (LRA), gives exceptional performances for several decades, thanks to its choice of thermal design and materials. Since 1969, NASA's Apollo 11, 14 and 15 missions, designed by a team led by C. O. Alley, D. Currie, P. Bender and Faller [2, 3], and the Soviet missions Luna 17 and 21, have deployed LRAs on the Moon surface, giving a perfect demonstration of functionality and performance.

The accuracy of laser ranging from Earth to Moon surface passive targets commonly reaches centimeter level. In recent years, many upgrades in observing technology and data modeling have improved the LLR. At this time, LLR is one of the best methods for achieving high-accuracy tests of GR, including tests of WEP and SEP, of Geodetic Precession, of time-variability in $G$, of PPN parameter $\beta$ and of the inverse-square law of gravity [4](J. G. Williams et al., 2008 \& M. Martini, Acta Polytechnica 53 Supplement, 2013).

The APOLLO (Apache Point Observatory Lunar Laser-ranging Operation) station is located at the Apache Point Observatory (APO) in southern New Mexico, at an altitude of $2800 \mathrm{~m}$. This is a unique position for the first-class APO $3.5 \mathrm{~m}$ telescope astronomical facility to perform a fundamental physics experiment $[\underline{5}$. Before the advent of the new apparatus of the APOLLO station, the accuracy limit in LLR measurements had been about $20 \mathrm{~mm}$. Now APOLLO seems to be able to provide $1 \mathrm{~mm}$ accuracy, though this is difficult to verify because the software models do not yet have sufficient precision.

The relative orientation of the Moon changes up to ten degrees during a month with respect to the line of sight from the earth station to the lunar array. 


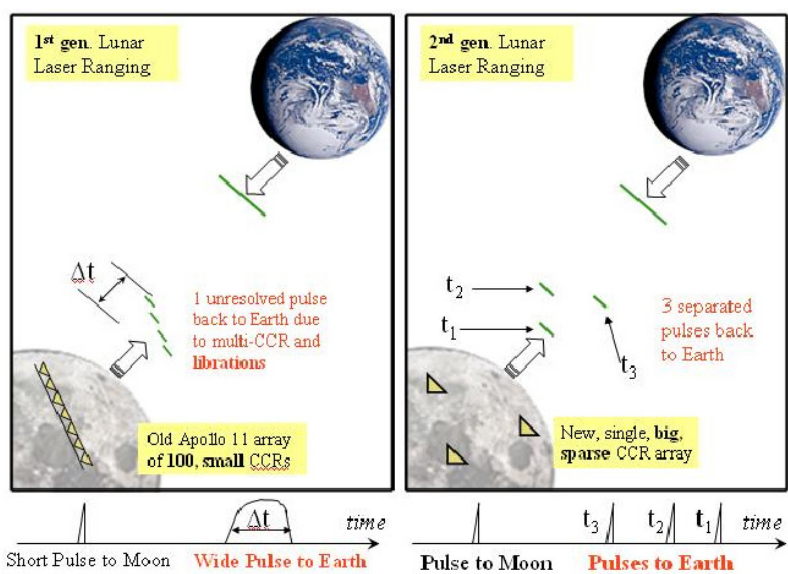

Figure 1. Concept of the 2nd generation of Lunar Laser Ranging.

This phenomenon is called "lunar libration", and it produces the primary range error. Considering the Apollo 15 array, the length, from corner to corner, is about $120 \mathrm{~cm}$, so librations generate a difference in distance between the extremes of $200 \mathrm{~mm}(120 \mathrm{~cm} \times$ $\left.10^{\circ}\right)$. This trivial geometrical calculation is shown for the first time by the APOLLO observations, confirmed by variation of the shape and full width of signal, as a function of the libration angle. The widest base, standing for corner-to-corner, is $1.1 \mathrm{~ns}$ (round-trip), or $165 \mathrm{~mm}$ one-way difference. Intrinsic to the multiCCR structure of the arrays, this causes an uncertainty widening of the return pulse, thus imposing a limit on the statistical error. This can add over $50 \mathrm{~mm}$ to the measurement error per photon, in a root-meansquare sense. It is by far the principal source of range uncertainty and, to achieve the millimeter level, one must collect thousands of photons.

\section{2ND Generation of LLR}

The general concept of the second generation of LLR is to consider a number (notionally eight) of large single Cube Corner Retroreflectors (CCRs). Each of these will produce a light echo that, with a single photoelectron detection system such as the current APOLLO system, can be used to improve the ranging beyond the limit of accuracy determined by the librational effects of current arrays and the laser pulse length. When single CCRs are used, the return is unaffected by the libration, that is, there is no increased widening of the FWHM caused by the librational effects and by the CCR itself. In this way, an accuracy improvement on the time of flight measurement of 1 ns (roundtrip) will be obtained. If two such single reflectors are deployed with a relative distance of tens of meters, their return of light will be recorded separately and can be recognized by comparison with the nominal orbit of the Moon and the rotational parameters of the Earth 6. This idea is illustrated schematically in Fig. 1 .
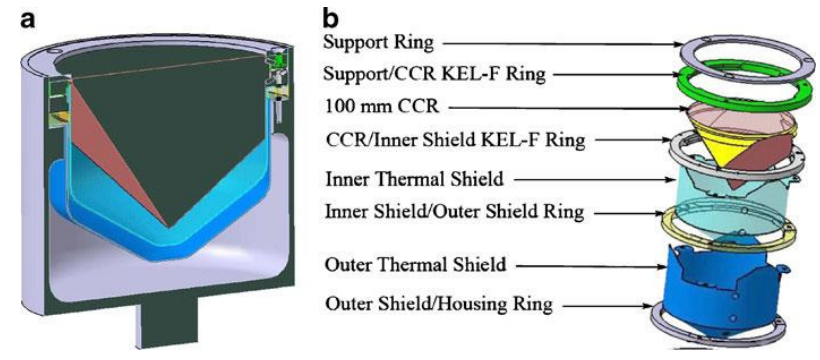

Figure 2. Section and exploded of the MoonLIGHT/ LLRA-21st CCR in its housing.

\section{The New Maryland/Frascati PAYLOAD}

The University of Maryland (UMD) and INFN-LNF are now proposing a new approach to the Lunar Laser Ranging Array (LLRA) technology, the experiment MoonLIGHT (Moon Laser Instrumentation for General relativity High-accuracy Tests), parallel to the LLRA-21st (Lunar Laser Ranging Array for the 21st Century) project, led by Douglas Currie [7. We currently use a $100 \mathrm{~mm}$ CCR realized in a special kind of glass, Suprasil 1 (formerly Suprasil T19), the same material as was used both in old LLRA and in LAGEOS (Laser Geodynamics Satellites). This will be mounted in an aluminum housing, thermally shielded from the lunar environment, in order to maintain a relatively constant temperature during the succession of lunar day and night. Moreover the CCR is also isolated from the housing by two coaxial "gold cans", to ensure that the CCR receives relatively little thermal input, as an effect of the low temperature of the lunar night and the high temperature of the lunar day. The mounting of the CCR inside the housing is shown in Fig. 2 For this mounting, one could use a ring of KEL-F, a special kind of plastic material that is already used in LAGEOS satellites, due to its good insulating, non-hygroscopic and low out-gassing properties. The CCR and its housing will be set by a rod into the lunar regolith to a depth of $1 \mathrm{~m}$ [8].

\subsection{Thermal and Optical Tests in FRASCATI}

SCF (Satellite/lunar laser ranging Characterization Facility), at LNF/INFN in Frascati, is a cryostat where we are able to reproduce the space environment: cold (77 K with Liquid Nitrogen), vacuum, and the Sun spectra. The SCF includes a Sun simulator ${ }^{1}$ that provides a $40 \mathrm{~cm}$ diameter beam with a close spectral match to the AM0 standard of 1 Sun in space $\left(1366.1 \mathrm{~W} / \mathrm{m}^{2}\right)$, with uniformity better than $\pm 5 \%$ over an area of $35 \mathrm{~cm}$ diameter. Next to the cryostat we have an optical table, where we can reproduce the laser path from Earth to the Moon, and back, studying the Far Field Diffraction Pattern (FFDP) coming back from the CCR to the laser station, which is useful for

\footnotetext{
1 ww.ts-space.co.uk
} 
understanding how good the optical behavior of the CCR is.

The SCF-Test 9 is a new test procedure, never performed before, for characterizing and modelling the detailed thermal behavior and the optical performance of laser retroreflectors in space for industrial and scientific applications. We perform an SCF-Test on the MoonLIGHT CCR to evaluate the thermal and optical performance in a space environment. For the thermal measurements we use both an infrared (IR) camera and temperature probes, which give real time measurements of all the components of the CCR and its housing. In particular we look at the temperature difference between the front face and the tip of the CCR, studying how the FFDP changes during the different thermal phases. This is the best representative of the thermal distortion of the return beam to the Earth. Various configurations and designs of the CCR and the housing have been tested and are being tested in the SCF Facility, with the solar simulator, temperature data recording, the IR camera and measurements of the FFDP [10].

\section{EXPERIMENTAL RESUlts}

Figure 4 shows the Moon LIGHT/LLRA-21st flight CCR FFDP intensity variation at Moon velocity aberrations $(2 \mathrm{~V} / \mathrm{c})$, during key points of the SCFTest: (1) in air, (2) in vacuum, (3) during cooling of the chamber's shields, (4) Sun on orthogonal to the CCR's face with the housing temperature controlled at $T=310 \mathrm{~K},(5) \mathrm{Sun}$ on at $30^{\circ}$ of inclination (no "break-through"), (6) Sun on at $-30^{\circ}$ of inclination ("break-through"), (7) Sun on orthogonal with the housing temperature left floating, (8) Sun off. From this graph we can deduce that the intensity decreases during non-orthogonal illumination of the CCR, in particular when the Sun enters the housing cavity during the "break-through" phase. This effect is due to a strong increase in the "Tip-Face" thermal gradient during this phase of the test. When the housing temperature is left floating, the intensity increases slightly because the "Tip-Face" gradient decreases.

Although, by default, this configuration of the MoonLIGHT/LLRRA-21st CCR seems to work in the right way, the first $\mathrm{SCF}$-test brought to light some important considerations that need to be explored, understood, and in many cases, improved. First of all, Fig. 3 clearly shows that a major loss of signal intensity, at the useful velocity aberration, is a consequence of the solar "break-through", when the CCR is illuminated in a non-orthogonal way. To limit this effect, we have designed and realized an aluminum "solar shade", which, mounted on the frontal ring of the housing, blocks the solar radiation during the "break-through" phase (Fig. 4).

The SCF-Test of this "Shade configuration" has already been done (from 24/03/2010 to $27 / 03 / 2010$ ), in the same way as the previous measurements. The use of "Solar Shade" has two additional important

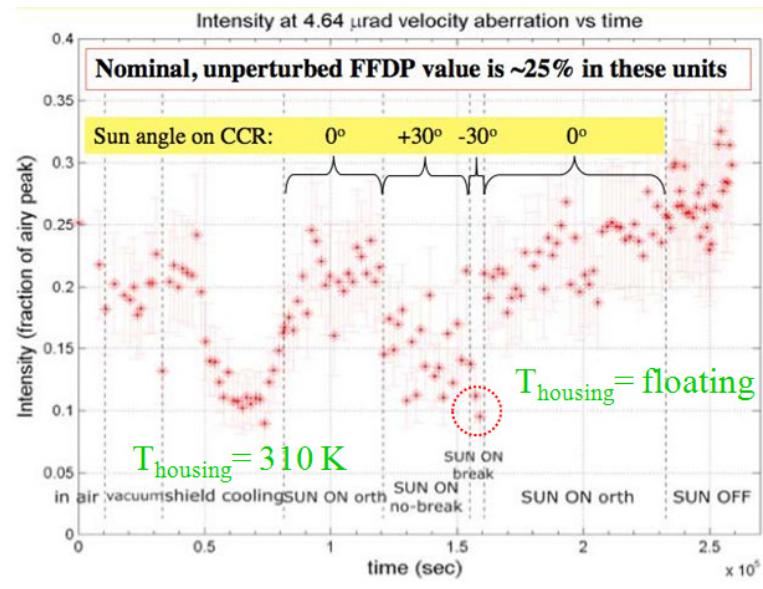

Figure 3. MoonLIGHT/LLRRA-21st flight CCR FFDP intensity variation.

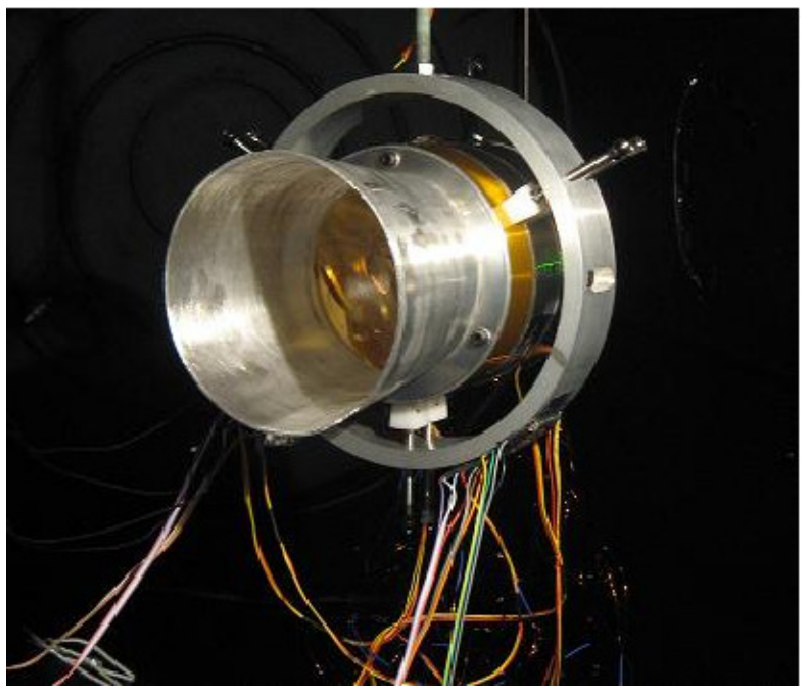

Figure 4. The MoonLIGHT/LLRRA-21st CCR in the "shade configuration", ready to be tested.

motivations. First of all, it acts as a protection from eventual dust deposition on the CCR frontal face, preventing a decline in the efficiency of the reflector in time. In addition, the shade provides protection from Ultra Violet light, which could change the optical properties of the reflector. Measurements of this kind will provide information on the CCR behavior in every phase of its lunar life, giving a complete and definitive characterization of the payload, which already seems to work quite well for our purposes. In this context, our MoonLIGHT/LLRRA-21st CCR, is becoming one of the most important candidates to be the laser retroreflector Core Instrument of the ILN project [11.

\section{Conclusions}

Lunar Laser Ranging remains one of the most powerful and competitive of all methods and technologies for investigations of this kind, and our work has been contributing to the development of the LLR, from several points of view. 
First of all, a new winning philosophy has been shown for a second generation of LLR, proposing a new prototype for a laser retroreflector. The experimental results indicate that this new payload can be a real candidate for improving the performance of LLR. In this regard, the ILN project offers a real opportunity to bring these new payloads on the lunar surface.

\section{ACKNOWLEDGEMENTS}

LLR gratefully acknowledges the major contribution to its work that has come from tight collaboration with the University of Maryland, personified by Douglas Currie, and from the INFN-LNF research group, led by Simone Dell'Agnello.

\section{REFERENCES}

[1] Dvali, G.; A.Gruzinov and M.Zaldarriaga, The Accelerated Universe and the Moon, Phys. Rev. D 68 024012, 2003

[2] Alley, C. O., et al. 1969, Laser Ranging Retroreflector, in Apollo 11: Preliminary Science Report (Tech. Rep. SP-214; Washington: NASA). 1970, Science, 167, 368

[3] Faller, J. E., et al. 1971, Laser Ranging Retroreflector, in Apollo 14: Preliminary Science Report (Tech. Rep. SP-272; Washington: NASA). 1972, Laser Ranging Retroreflector, in Apollo 15 Preliminary Science Report (Tech. Rep. SP-289; Washington: NASA)

[4] J.G. Williams, S. Turyshev and D.H. Boggs, Progress in Lunar Laser Ranging Tests of Relativistic Gravity, Phys. Rev. Lett.93.261101 (2008)
[5] T.W. Murphy, E.G. Adelberger, J.B.R. Battat et al., APOLLO: the Apache Point Observatory Lunar Laser-ranging Operation: Instrument Description and First Detections, Publ.Astron.Soc.Pac.120:20-37, 2008

[6] S. Dell'Agnello et al., IEEE Aerospace Conference, Next Generation Lunar Laser Ranging and its GNSS Applications, Big Sky (MT), USA, 2010, and references therein

[7] Currie D.G. et al., A Lunar Laser Ranging Retro-Reflector Array for NASA's Manned Landings, the International Lunar Network, and the Proposed ASI Lunar Mission MAGIA, 16th International Workshop on Laser Ranging Instrumentation, October 13-17, 2008

[8] D.Currie et al., A Lunar Laser Ranging Retroreflector Array for the 21st Century, "Acta Astronautica" 68 (2011) 667-680

[9] S.Dell'Agnello et al., Creation of the new industry-standard space test of laser retroreflectors for the GNSS and LAGEOS, Galileo Issue in Journal of Advances in Space Research, Scientific application of Galileo Navigation Satellite System, 47 (2011) 822-842

[10] A. Boni et al., Optical far field diffraction pattern test of laser retroreflectors for space applications in air and isothermal conditions at INFN-LNF. INFN-LNF Report LNF-08/26(IR), 2008

[11] Dell'Agnello et al, International Lunar Network Core Instruments Working Group, Final Report Study Period: June 2008 to July 2009, and references therein (http://iln.arc.nasa.gov 